\title{
Epistémologie du « sujet enseignant », dans son rôle d'évaluateur de compétences
}

\section{Noël Cordonier}

\section{(2) OpenEdition}

\section{Journals}

Édition électronique

URL : http://journals.openedition.org/ripes/513

DOI : 10.4000/ripes.513

ISSN : 2076-8427

Éditeur

Association internationale de pédagogie universitaire

Référence électronique

Noël Cordonier, «Epistémologie du « sujet enseignant », dans son rôle d'évaluateur de compétences », Revue internationale de pédagogie de l'enseignement supérieur [En ligne], 27(2) | 2011, mis en ligne le 16 janvier 2012, consulté le 08 septembre 2020. URL : http://journals.openedition.org/ripes/513 ; DOI : https://doi.org/10.4000/ripes.513

Ce document a été généré automatiquement le 8 septembre 2020

Article L.111-1 du Code de la propriété intellectuelle. 


\title{
Epistémologie du « sujet enseignant ", dans son rôle d'évaluateur de compétences
}

\author{
Noël Cordonier
}

\section{Introduction}

1 Le lecteur de ces pages est invité à se méfier de sa bienveillance : l'intérêt qu'il pourrait y prendre n'est sans doute dû qu'au profil de leur auteur, qui n'est pas un spécialiste des sciences de l'éducation, mais un didacticien surtout habitué aux méthodes textuelles et historiques. Mon ambition ne peut donc être autre que de lire un référentiel de compétences estimé prototypique et d'observer les acteurs qui l'ont adopté, les enseignants. Puis de poser des questions élémentaires : quelles conceptions, quelles images du sujet enseignant ce référentiel de compétences infère-t-il, en général ou, de manière plus spécialisée, lorsqu'il prie les formateurs d'évaluer les compétences de leurs étudiants? Bien qu'il s'agisse d'un costume trop grand pour elle, ma contribution s'inscrit néanmoins en épistémologie. Elle cherche à dégager, sous la multiplicité des tâches et des injonctions qui sont assignées aux enseignants que nous sommes, quelques éléments du socle philosophique sur lequel reposent nos gestes et nos discours ${ }^{1}$.

Concrètement, cette étude procède d'une analyse de corpus et de textes (un référentiel de compétences, une grille d'évaluation) et d'une brève analyse de pratiques (un dispositif de certification). Mes techniques de recherche sont celles de la linguistique (étude des discours et des langages) et de la sémiologie (étude des systèmes de signification). Chemin faisant, je solliciterai également l'histoire de l'éducation et celle des idées. Une remarque encore: dans ma discipline de base, les lettres, suite aux travaux de Paul Ricoeur, Jerome Bruner, Gareth Morgan, ... on considère la métaphore comme un mode de connaissance particulier mais patenté. Je me permettrai donc, ici ou là, des tours imagés. 
Quand, en 2005, bien après le Québec et de nombreuses autres institutions de formation ${ }^{2}$, la Haute école pédagogique (ci-après, HEP) du canton de Vaud a adopté son référentiel de compétences, ses motivations ont été semblables à celles de ses prédécesseurs : un tel cadre serait de nature, nous disait-on, à faire évoluer l'activité enseignante de la conception artisanale du "métier " vers celle de la "profession", caractérisée par une cartographie précise et complète des activités à savoir conduire. Même si les termes de ce qui a été présenté comme une modernisation certaine sont largement connus, il m'importe de les rappeler (cf. Tableau 1), parce que j'aurai besoin de cette histoire de l'enseignement, de cette liste d'oppositions binaires et de ces mots pour situer mes observations.

Tableau 1 : « deux figures professionnelles », selon Astolfi (2005).

\begin{tabular}{|l|l|}
\hline Métier & Profession \\
\hline Transmission & Construction \\
Instruction & Formation \\
Maître & Médiateur \\
Elève & Apprenant \\
Programme & Curriculum \\
Leçon, cours & Dispositif \\
Notion & Concept \\
Mémoire & Cognition \\
Connaissances & Compétences \\
Contrôle & Evaluation \\
Transmission [bis] & Instruction \\
\hline
\end{tabular}

4 Après cinq ans de pratiques sous le nouveau régime de la "profession ", il paraît nécessaire d'entamer une évaluation du parcours. Nos questions de recherche seront donc celles-ci : Comment le sujet enseignant, tel qu'il est prescrit ou défini par ce référentiel, est-il concrètement endossé par les formateurs de la HEP ? Quels sont les points forts et les points faibles de ce nouveau régime? Et qu'est-ce qui, de ce bilan, pourrait être utile à d'autres institutions?

5 Etant donné l'impossibilité de commenter par le menu toutes les activités, habiletés ou autres savoirs préconisés dans un référentiel, on n'observera ici, pour ce premier examen, que les deux qualités les plus emblématiques, les plus à même de caractériser « la nouvelle figure du maître [...] : celle de l'expert [...] et celle de l'innovateur » (JacquetFrancillon, 2005, p. 110).

\section{L'innovateur}

6 En prélevant dans le référentiel de la HEP du canton de Vaud l'énoncé de la compétence qui enjoint l'enseignant d'innover, j'ai pour principal objectif de vérifier, par l'histoire 
de l'éducation, combien et comment cette tâche induit une conception effectivement nouvelle du sujet enseignant. A cette fin, les travaux de Vincent Monetti sont précieux pour dérouler un bout de son histoire récente. Ne retenant ici de la complexe définition de l'innovation que des traits succincts, à savoir qu'elle ne se résume pas à des contenus, à des objets mais qu'elle est aussi « symbolique et culturelle (Monetti, 2002, p.18), et qu'elle varie sensiblement selon les contextes, nous chercherons surtout à rappeler les rôles différents qui ont été confiés aux enseignants lors des diverses phases de son histoire ${ }^{3}$. Selon Monetti, dont les observations du cas français semblent valoir pour l'ensemble de la francophonie, l'innovation scolaire récente a connu trois temps.

\subsection{0 - 1980 : L'enseignant, agent de l'innovation}

7 Répondant aux nécessités de la démocratisation des études, dans un contexte caractérisé par l'essor et les espoirs du structuralisme, lequel a souvent engendré des modèles didactiques de type applicationniste, l'innovation de ces années est du monopole de l'Etat. Associée aux notions de réforme ou de changement planifié, cette forme d'innovation est plutôt normative. Ainsi en est-il allé, dans bien des pays, de l'introduction des mathématiques modernes ou de ce qui, sous différentes appellations, a concerné le français dit renouvelé. Ce mode centralisé et descendant, a fait de l'enseignant un agent de l'innovation, après qu'il eut été, dans la plupart des cas, dûment « recyclé » ou formé aux nouvelles méthodes. L'enseignant ne produisait donc pas de l'innovation, il l'appliquait.

\subsection{0 - 1990 : l'enseignant, acteur de l'innovation}

8 Dès la fin des années 70, les Etats ont peu à peu renoncé à monopoliser le changement et ils ont cédé une partie de l'initiative aux établissements scolaires, au «terrain ». En France, par exemple, la création des Zones d'Education Prioritaires (ZEP) a engagé ceux-ci à lancer des projets pédagogiques innovants et c'est également le moment de la rénovation des collèges et de la création des Fonds d'aide à l'innovation. En Suisse romande, lorsque du matériel d'enseignement a été conçu par des conseillers pédagogiques œuvrant pour l'Etat, les enseignants du terrain y ont souvent participé activement, via des représentants ou des commissions. Bien que, dans cette phase, l'Institution étatique contrôlât la novation, celle-ci était à la fois descendante et émergeante, ce qui tendait à ne plus considérer l'enseignant comme un simple agent fonctionnel, mais comme un acteur, engagé dans un processus et doté d'une force de proposition et de négociation.

\subsection{De 1990 à aujourd'hui : l'enseignant, créateur d'innovation}

Même si la troisième phase délimitée par Vincent Monetti s'arrête au tournant du millénaire, elle nous semble conserver toute son actualité aujourd'hui encore, ce d'autant plus que les Etats, qui ont poursuivi l'application des recettes néolibérales et dégraissé bien des services publics (conseillers pédagogiques, éditions scolaires, ...), ont souvent renoncé, ces dernières années, à endosser ou promouvoir des politiques scolaires explicites. 

l'innovation ne provient plus d'«en haut», plus du dehors de l'école, ni même de l'établissement scolaire, mais elle est endogène, déposée en chaque enseignant. La capacité individuelle d'innovation devient donc un facteur essentiel du changement, ainsi que l'expriment clairement la compétence $\mathrm{n}^{\circ} 2$ de la HEP vaudoise et sa $4^{\mathrm{e}}$ composante.

11 L'enseignant doit «s'engager dans des démarches d'innovation ou de recherche pour enrichir sa pratique professionnelle ». Il « initie ou participe aux projets pédagogiques conduits dans la classe ou dans l'établissement. Ceux-ci constituent en effet des leviers majeurs de développement des connaissances professionnelles. L'enseignant contribue également à des projets de recherche qui visent à l'amélioration des pratiques d'enseignement » (HEP du canton de Vaud, pas de date).

Assurément, le professionnel est le créateur, l'auteur de l'innovation, ce qui donne lieu à des initiatives et des entreprises de recherche et d'enseignement autonomes, originales et variées.

\subsection{Un déplacement de majuscule}

13 Même très raccourci, cet historique de l'innovation (dans son sens large) suffit à mesurer un déplacement de majuscule. Celle qui était posée jusque dans les années 80 environ sur l'Etat souverain, prescripteur d'innovation, se retrouve maintenant diffractée sur chaque enseignant. Celui-ci, qui était un sujet dépendant et soumis, est désormais estimé apte à créer de l'innovation. Sur le papier à tout le moins, la responsabilité a changé de camp, elle est maintenant intériorisée, individuelle. Autonome, considéré tout autant capable de diagnostiquer les besoins de changement et de novation qu'à les combler, le sujet enseignant est également responsable de la conception et de la mise en place des actions de recherche ou de formation qui en découlent. Ajoutant une illustration à celles déjà bien connues, on confirme ainsi que l'instituteur d'autrefois, qui agissait de manière déléguée au nom d'une autorité plus grande que lui, a effectivement été remplacé par le professionnel, doté, en théorie, d'une autorité et d'une réflexivité propices à l'anticipation, la recherche et l'action. Enfin, si l'on mesure ce mandat à la nouvelle grille des notions caractérisant la profession, on remarque que le rapport de l'enseignant à la temporalité a changé. Du métier à la profession, le rôle du passé et de la tradition, qu'incarnait encore l'Etat des années soixante, est désormais moins important que celui du présent. Hier, l'Etat se donnait peu ou prou pour mission d'assumer la pérennité du système et les innovations étaient souvent à tendance normative ou régulatrice, fondées sur des valeurs idéologiques ou spirituelles déposées dans le passé. A l'inverse, aujourd'hui, «l'innovation devient un impératif de fonctionnement» (Monetti, 2002, p. 73), elle est une réponse sans cesse réajustée, flexible, mouvante aux observations que l'enseignant prélève quotidiennement, dans le hic et nunc de la relation pédagogique.

\section{L'expert évaluateur}

14 Après avoir évoqué l'innovateur tel qu'il est défini sur le papier, c'est-à-dire dans le texte du référentiel de compétences, examinons en acte, en situation réelle, sa seconde caractéristique importante, celle de l'expert ${ }^{4}$. Comment, dans les faits, «évalu[ons-

Revue internationale de pédagogie de l'enseignement supérieur, 27(2) | 2011 
nous] la progression des apprentissages et le degré d'acquisition des connaissances et des compétences des élèves/étudiants" (HEP du canton de Vaud, pas de date)? Comment, plus précisément encore, répondons-nous aux prescriptions qui nous intiment que «l'évaluation des performances se fonde sur des critères, méthodes et instruments rigoureux, permettant de mesurer des caractéristiques précises retenues comme indicateurs explicites des acquisitions » (id.) ? Parmi la bigarrure des formes et manières d'évaluer que la HEP expérimente depuis l'introduction de son référentiel de compétences, je n'en retiendrai ici qu'une seule qui me paraît exemplaire de notre sérieux investissement. Cette démarche a été présentée et finement commentée par l'un de ses concepteurs, mon collègue Luc-Olivier Bünzli ${ }^{5}$, qui a décrit une évaluation sommative du module interdisciplinaire Certifier dans la scolarité / Diversifier en maths, suivi par les étudiants de $3^{\mathrm{e}}$ année de la filière d'enseignement préscolaire/primaire de la HEP (cf. Tableau 2). Je ne juge pas et je n'évalue pas cette évaluation ; j'essaie de la lire avec les moyens déjà évoqués, qui vont de la sémiologie - la forme même de la grille - à la mise en évidence de ses implicites et de l'outillage conceptuel qu'elle convoque. J'insiste: cette grille n'est étudiée ici que du point de vue sémiologique, comme une «forme-sens » où tout est signifiant, de la mise en page au lexique, ou aux champs sémantiques. Dans cette perspective, il ne sera pas nécessaire de décrire par le menu les modalités de son emploi.

Ce qui frappe d'abord, dans une telle construction, c'est la multiplicité des critères, la conjugaison des trois regards experts, le libellé minutieux des items. Cette grille rigoureuse aidera trois correcteurs à évaluer, chacun selon leur spécialité, une épreuve rédigée à huis clos, en 4 heures, par un candidat, puis à mettre en commun leurs appréciations. En absolu, ne sommes-nous pas, avec un tel instrument, proches d'une objectivité quasi maximale, lorsqu'il s'agit d'évaluer des habiletés et connaissances dans le domaine des sciences humaines? A cette volonté d'objectivité s'ajoute celle de l'exhaustivité, qui se manifeste aussi bien dans les intitulés de plusieurs items (par exemple, "tous les éléments ", "toutes les étapes ", "tous les liens ») que par le fait que la grille, par définition, combine une lecture verticale (celle de chaque correcteur) et une lecture transversale, par thèmes communs aux trois correcteurs. 
Tableau 2 : grille d'évaluation, version 2008 , d'un module interdisciplinaire à la HEP du canton de Vaud : didactique des mathématiques, évaluation et pédagogie spécialisée

\begin{tabular}{|c|c|c|c|}
\hline $\begin{array}{l}\text { Regards / } \\
\text { Critères }\end{array}$ & $\begin{array}{l}\text { Élaboration d'un test évaluant } \\
\text { une compétence dans le champ } \\
\text { conceptuel de la multiplication } \\
\text { (Compétence 4) }\end{array}$ & $\begin{array}{l}\text { Argumentation de } \\
\text { l'adéquation du test en } \\
\text { lien avec une } \\
\text { évaluation de } \\
\text { compétences dans le } \\
\text { champ conceptuel de la } \\
\text { multiplication } \\
\text { (Compétence 5) }\end{array}$ & $\begin{array}{l}\text { Regard réflexif sur le test en } \\
\text { lien avec les difficultés } \\
\text { susceptibles d'être } \\
\text { rencontrées par les élèves } \\
\text { (Compétence } 7 \text { ) }\end{array}$ \\
\hline & Expert 1 & Expert 2 & Expert 3 \\
\hline Pertinence & $\begin{array}{l}\text { 1) La compétence, les critères, la } \\
\text { tâche et les indicateurs sont en } \\
\text { lien avec le champ conceptuel } \\
\text { défini. } \\
\text { 2) La compétence visée est } \\
\text { identifiée et précisée au travers } \\
\text { de critères. } \\
\text { 3) Les indicateurs sont précisés en } \\
\text { lien avec chaque critère ( } 3 \text { au } \\
\text { minimum). } \\
\text { 4) Ily a adéquation entre niveau } \\
\text { scolaire et complexité du test. }\end{array}$ & $\begin{array}{l}\text { 1) Les liens entre les } \\
\text { éléments sont mis en } \\
\text { évidence: } \\
\text { a. compétence et } \\
\text { critères, } \\
\text { b. critères et } \\
\text { indicateurs, } \\
\text { c. critère et échelle } \\
\text { 2) La complexité du test } \\
\text { est identifiée. } \\
\text { 3) L'argumentation se } \\
\text { référe à des cadres } \\
\text { théoriques. }\end{array}$ & $\begin{array}{l}\text { 1) Des difficultés susceptibles } \\
\text { d'être rencontrées par les } \\
\text { élèves sont identifiées et } \\
\text { discutées. } \\
\text { 2) La discussion fait référence } \\
\text { aux contenus développés } \\
\text { dansle cours. } \\
\text { 3) Des liens avec les textes } \\
\text { proposés en lecture sont } \\
\text { réalisés. }\end{array}$ \\
\hline $\begin{array}{l}\text { Correction } \\
\text { (exactitude) }\end{array}$ & $\begin{array}{l}\text { 1) Le choix de la compétence } \\
\text { visée est correct en fonction du } \\
\text { Plan d'études vaudois. } \\
\text { 2) La tâche recouvre le champ } \\
\text { conceptuel défini. } \\
\text { 3) Les outils mathématiques sont } \\
\text { corrects parrapport au système } \\
\text { de numération. }\end{array}$ & $\begin{array}{l}\text { 1) Les références } \\
\text { théoriques sont } \\
\text { exactes. } \\
\text { 2) Les liens entre les } \\
\text { différents éléments } \\
\text { sont corrects. } \\
\text { 3) L'argumentation est } \\
\text { exacte. }\end{array}$ & $\begin{array}{l}\text { 1) Les liens entre les difficultés } \\
\text { et la tâche sont adéquats. } \\
\text { 2) L'argumentation proposée } \\
\text { est convaincante dans son } \\
\text { ensemble. } \\
\text { 3) Les références théoriques } \\
\text { sont exactes. }\end{array}$ \\
\hline
\end{tabular}

Si l'on déduit de ce premier regard sur la grille que l'examiné (le candidat à l'enseignement) a toutes les chances d'être évalué au plus près de ce qu'il a produit et en référence étroite avec les objectifs explicites du module et la notion de compétences, on ne peut s'empêcher de s'interroger sur le professeur, sur le sujet enseignant exerçant ici son rôle d'évaluateur. De quoi une telle sophistication, une telle minutie et une telle conjugaison des regards sont-elles aussi les indices?

Les critères qui cherchent à mesurer minutieusement, en tranches finement découpées, l'adéquation ou l'écart des réponses du candidat par rapport aux objectifs annoncés du module, qui visent à confirmer, en d'autres termes, le trajet plus ou moins réussi de l'input à l'output, mettent d'abord en évidence, dans le geste même où ils tendent à minimiser l'intromission subjective de l'examinateur, la primauté de la raison instrumentale.

Le philosophe Charles Taylor définit la raison instrumentale comme "[...] cette rationalité que nous utilisons lorsque nous évaluons les moyens les plus simples de parvenir à une fin donnée. L'efficacité maximale, la plus grande productivité mesurent sa réussite » (Taylor, 2007, p. 15). Ici, la raison instrumentale est celle qui s'est attelée à découper des segments de sens nettement délimités, pertinents, et à les mesurer, pièce par pièce, en se référant aux compétences définies par ce module interdisciplinaire que nous avons examiné. Autrement dit, le vœu aussi intense d'objectivité et la recherche de connaissances ou de savoir-faire si minutieusement définis tendent, d'une part, à chosifier le savoir observable et, d'autre part, à favoriser des mécanismes idéalement impersonnels d'observation. Le type de rationalité qui est à l'œuvre dans cet exercice d'évaluation est alors, effectivement, un "[...] mode de connaissance empirique, formalisée et utilitaire » (Mongeau, 1978). 

d'efficience, une telle grille est à proprement parler un dispositif, qu'on se contentera de définir, pour l'instant, à partir de son seul sens courant: un mécanisme de pièces disposées en vue d'une exploitation réussie. Ce dispositif réduit la raison de l'évaluateur à sa dimension instrumentale. Il y a lieu de s'interroger plus avant à ce sujet.

Il nous importe davantage de sonder le sujet expert lui-même, celui dont une des tâches est d'évaluer. $\mathrm{Si}$, indubitablement, il s'est donné un instrument hautement perfectionné, un dispositif fonctionnant quasi sans son intromission subjective, ne fautil pas aussi soupçonner, en creux, une certaine réticence, voire même une difficulté à assumer la responsabilité d'un jugement, d'une évaluation? Le nombre d'évaluateurs, le recours à une raison restreinte à sa partie instrumentale ou encore la rassurante évidence de l'extériorité quasi autonome que constitue la grille ne trahiraient-ils pas la crainte d'endosser ce qui, dans une évaluation, relève de la subjectivité... du sujet enseignant? Voire même la crainte à affronter la subjectivité de l'évalué ? Autrement dit, la forte instrumentalisation de la grille ne trahirait-elle pas, en creux, un manque de confiance du sujet enseignant ainsi qu'une certaine « désubjectivisation » du sujet, une certaine dépersonnalisation, volontaire ou pas? Et ne traduirait-elle pas, en sus, le poids d'une institution qui, par le référentiel même qu'elle a retenu, vise l'évaluation la plus objective?

\section{Interprétation des résultats : forces et faiblesses du sujet postmoderne}

Même si on laisse à cette dernière question sa fragilité d'hypothèse et sa dimension prospective, on se trouve en peine quand on tente de rapprocher les deux facettes constitutives de l'enseignant professionnel que nous avons séparées le temps de l'analyse, celle de l'innovateur et celle de l'expert (ici dans sa tâche d'évaluateur). D'une part, le professionnel tel que défini par le référentiel de compétences se voit doté d'une forte autonomie cognitive, conceptuelle, analytique et active dans la mise en place de dispositifs : c'est brosser le portrait de l'expert-innovateur. Mais, d'autre part, la mise en place de dispositifs rigoureusement réglés par la seule raison instrumentale attesteraient une difficulté du sujet expert-innovateur à endosser, à assumer complètement la subjectivité de son rôle, en tous les cas pour ce qui concerne l'évaluation des apprentissages.

Ainsi qu'en témoignent les protocoles d'expertises, l'enseignant, sous l'idéal évidemment noble et indiscutable de viser à l'évaluation la plus rigoureuse, se prive d'un usage plus large et plus complet de la raison et, peu ou prou, il disparaît sous des dispositifs sophistiqués : le sujet expert d'aujourd'hui (toujours rapporté ici à sa tâche d'évaluateur) jouit d'une liberté nouvelle par rapport aux enseignants des générations précédentes, mais il est peut-être aussi caractérisé par un manque de confiance dans son jugement et par une restriction de sa raison à sa seule dimension pragmatique. Georgio Agamben, qui a récemment proposé une archéologie du concept de dispositif, $\mathrm{du}$ sens qu'il avait en théologie à celui que lui a donné Michel Foucault, estime que les dispositifs (des plus concrets, tels les ordinateurs ou les téléphones portables aux plus généraux, qui ont rapport avec des formes de pouvoir, comme la justice ou l'école)« [...] déterminent ce que le sujet devient par le type d'usage qu'il en fait» (Zarka, 2008).

Revue internationale de pédagogie de l'enseignement supérieur, 27(2) | 2011 
Selon lui, un dispositif désigne «[...] ce en quoi et par quoi se réalise une pure activité de gouvernement sans le moindre fondement dans l'être " (Agamben, 2007, p. 26-27).

Posée sur l'histoire de l'épistémologie occidentale, ce sujet à la fois libre et faible, ou restreint, semble vérifier la prégnance et le règne du paradigme postmoderne. En effet, ainsi que nous l'a montré l'historique de l'innovation depuis les années soixante, le Tiers Garant, la caution que représentait l'Etat s'est délitée. L'Etat incarnait et portait l'Histoire, et avec elle une conception du temps selon laquelle le passé de la civilisation créait un projet, une téléologie, un avenir certes incertain, mais néanmoins riche d'une certaine positivité, d'une finalité, que celle-ci relève des anciennes transcendances historiques ou, depuis Kant et les Lumières, $d u$ «transcendental $»^{6}$. Sous le régime de l'Etat et sous ce que celui-ci emblématisait, l'individu était fortement encadré par le système : sujet minuscule, soumis, - aliéné, disait le marxisme - mais sujet pris dans la tension entre les régulations qu'incarnait l'Etat et les dérégulations alimentées par la volonté de réalisation individuelle. Aujourd'hui, consécutivement à l'affaissement des idéologies, à la fin de l'Histoire et des Grands Récits fondateurs (ceux des religions, des idéologies, de la science), le sujet enseignant se retrouve doté d'une liberté et d'une autonomie inédites. Il n'est plus le simple agent d'une chaîne de transmission, mais à tout instant impliqué et interpellé par un présent labile, indéfinissable, il est en posture de le décrire et de l'infléchir par des actions locales, ponctuelles. Il est un sujet innovateur.

Pour ne considérer que les plans de l'école et de l'éducation, cette liberté et cette autonomie ont toutefois un coût, que Lyotard, avait prophétiquement repéré dans le rapport sur le savoir qu'il rédigea en 1979 pour le conseil des Universités à la demande du gouvernement québécois : «Dans le contexte de la délégitimation, [suite à la fin des grands récits spéculatifs et d'anticipation], les universités et les institutions d'enseignement supérieur sont désormais sollicitées de former des compétences, et non plus des idéaux » (Lyotard, 1979, p. 79).

Pour une part, les référentiels de compétence dotent le sujet enseignant d'une forte capacité d'expertise, d'évaluation, d'action, d'initiative. Impliqué comme tout un chacun dans un présent véritablement ouvert et sans lignes directrices, l'enseignant innovateur sait amener à l'école ce dont elle a besoin pour ses permanentes régulations. D'une certaine manière, il crée l'école et le réel par les incessants ajustements qu'il apporte.

Mais cette liberté et cette autonomie trahissent aussi une certaine faiblesse. En régime classique ou moderne, les actions d'un sujet et notamment du sujet enseignant étaient garanties par un usage sans doute plus posé, plus accompli de la raison. Qu'il s'agisse de la conception idéaliste ancienne qui fondait une ontologie de la raison ou que celle-ci fût liée au transcendantal kantien, dans un tel régime, «[...] toutes les fonctions théoriques et pratiques de l'esprit - l'art, la science, la philosophie, la religion, l'éthique, le politique, la technique - sont traversées par le logos, participent à cette structure rationnelle d'où émergent les significations et les valeurs » (Mongeau, 1978).

Sur le cas limité que nous avons retenu, celui de l'évaluation, les référentiels de compétence pourraient donc témoigner d'une certaine abdication à exercer un usage plus ambitieux de la raison et de l'innovation. Ces référentiels tendent en effet à limiter la rationalité du sujet enseignant à un mode de connaissance empirique, formalisé et utilitaire. Un tel usage pragmatique de la raison fait donc exactement la force et la faiblesse du sujet enseignant: on l'invite à créer-utiliser l'instrument le plus apte à 
l'action (la raison instrumentale), mais on en rétrécit l'ambition et la portée (réduction du logos à la sa fonction pragmatique). Le geste d'évaluer se traduit, se concrétise par des dispositifs précis, mais la sophistication de ceux-ci semble dans le même temps l'indice d'un renoncement à assumer pleinement et la responsabilité de ce geste et le face à face avec la subjectivité de la personne évaluée.

\section{Conclusion pragmatique}

Passons, pour conclure le propos et, simultanément, pour élargir la question de recherche, à un cas concret, qui provient à nouveau de l'expérience en cours à la HEP vaudoise, mais qui a eu ou qui peut avoir des équivalents ailleurs. Si le rôle fort et responsable de l'enseignant-innovateur est illustré par la liste des innombrables activités de recherches appliquées et de développement en cours, ce qui pourrait trahir sa faiblesse, c'est-à-dire la « désubjectivisation » que peuvent entraîner les référentiels de compétences, s'observe dans la façon qu'a la HEP vaudoise de "gérer " les examens et plus exactement les cas d'échecs aux certifications. Aujourd'hui, ces cas semblent surtout régis par les seules règles et logiques du dispositif, que nous avons caractérisé par son autonomie et par ce que celle-ci entraîne, à savoir une certaine mise à l'écart des subjectivités, voire une certaine peur de la subjectivité assumée.

Le cas d'un étudiant qui se trouve en échec selon les modalités du règlement d'études vaudois est réglé par une machine administrative qui n'implique à aucun moment la considération de son dossier par l'ensemble des évaluateurs, par leurs subjectivités conjuguées et en dialogue. Autrement dit, il n'est prévu aucune conférence d'examen ; aucune commission n'a été jusqu'à présent constituée pour évaluer et statuer sur la personne en situation d'échec. En conséquence, quand l'ultime occurrence de se présenter à un examen a été tentée et perdue, celle-ci porte l'échec du parcours global du candidat, sans qu'une instance quelconque ne soit mandatée pour considérer le cas.

Simple carence d'un règlement et d'une procédure en rodage ? C'est l'hypothèse la plus rassurante. L'autre hypothèse, plus inquiétante, c'est que l'enseignant évaluateur se décharge sur l'appareil qu'il a conçu. Là, précisément, se trouve le risque de désinvestir les subjectivités, de renoncer à assumer le difficile mais indispensable usage d'une raison entendue dans la totalité de ses dimensions, comme un principe de régulation instrumentale et pragmatique, certes, mais aussi comme exercice pleinement endossé du jugement subjectif, compris à la fois comme un principe de tensions, de négociation, ainsi que de perfectibilité et de régulation morale. Autrement dit, le risque est fort de céder aux facilités d'une gestion taylorisée, émiettée et, selon nous, déshumanisée de la responsabilité.

\section{BIBLIOGRAPHIE}

Agamben, G. (2007). Qu'est-ce qu'un dispositif? Paris : Rivages Poche. 
Astolfi, J.-P. (2005). Le métier d'enseignant entre deux figures professionnelles. Repéré le 20 janvier 2010 à http://francois.muller.free.fr/diversifier/30compet.htm.

Bünzli, L.-O. (2010). Evaluer des compétences en formation : un défi. Dans L. Bélair, C. Lebel, N. Sorin, A. Roy \& L. Lafortune (Eds), Régulation et évaluation des compétences en enseignement, Vers la professionnalisation (pp. 253-265). Sainte Foy : Presses de l'Université du Québec.

Charlier, B., \& Peraya, D. (Eds) (2003). Technologie et innovation en pédagogie. Dispositifs innovants de formation pour l'enseignement supérieur. Bruxelles : De Boeck.

Cordonier, N. (2010a). L'épistémologie du sujet enseignant tel que construit par les référentiels de compétence et ses effets sur la culture scolaire. Dans H. Akefli \& S. El Harmassi (Eds.), Culture scolaire et formation des enseignants (pp. 8-24). Cahiers de l'Ecole Normale Supérieure de Meknès, Revue pédagogique et culturelle, No. 15, février.

Cordonier, N. (2010b). L'épistémologie du "sujet enseignant", tel qu'il apparaît dans les référentiels de compétences et les activités d'expertise. Dans L. Bélair, C. Lebel, N. Sorin, A. Roy \& L. Lafortune (Eds), Régulation et évaluation des compétences en enseignement, Vers la professionnalisation (pp. 239-252). Sainte Foy : Presses de l'Université du Québec.

Dufour, D.- R. (2008). Le divin marché, La révolution culturelle libérale. Paris : Denoël. Jacquet-Francillon, F. (2005). Les mutations récentes de la culture scolaire. Dans F. JacquetFrancillon, \& D. Kambouchner (Eds), La crise de la culture scolaire (pp. 103-113), Paris : Presses Universitaires de France.

Haute Ecole pédagogique du canton de Vaud (s.d.). Référentiel de compétences. Repéré le 28 novembre 2011 à http://www.hepl.ch/files/live/sites/systemsite/files/interfilieres/referentielcompetences-hep-vaud.pdf.

Jacquinot-Delaunay, G., \& Fichez, E. (Eds). (2008). L'université et les TIC. Chronique d'une innovation annoncée. Bruxelles : De Boeck.

Lyotard, J.-F. (1979). La condition postmoderne. Paris : Minuit.

Martinet, M. A., Raymond, D., \& Gauthier, C. (2001). La formation à l'enseignement, Les orientations, Les compétences professionnelles. Gouvernement du Québec, Ministère de l'Education.

Monetti, V. (2002). Etat des lieux : qu'est-ce que l'innovation en éducation ? L'exemple de la France depuis les années 1960. Dans V. Monetti (Ed.), Certitudes et paradoxes de l'innovation, Etat des lieux, états d'esprit (pp. 17-79). Paris : INRP.

Mongeau, Y. (1978). Libérer la démocratie. In L'encyclopédie de l'Agora (dossier « Raison ») Repéré le 28 novembre 2011 à http://agora.qc.ca/Documents/Politique--

Liberer_la_democratie_par_Yves_Mongeau.

Perrenoud, P. (1994). Former les enseignants primaires dans le cadre des sciences de l'éducation : le projet genevois. Recherche et Formation, 16, 39-60.

Taylor, C. (2007). Grandeur et misère de la modernité. Montréal : Editions Bellarmin.

Zarka, S. (2008). L'erreur du dispositif. Repéré le 20 janvier 2010 à http:// ingenieurdusymbolique.fr/4595. 


\section{NOTES}

1. Cet article découle en partie de la recherche « HEP 2008/04 » intitulée « Quelles perceptions et quelles connaissances des savoirs à enseigner et de leurs finalités en littérature française, de l'Université à l'entrée dans la profession enseignante au Secondaire 2 ? ». Ma contribution est parallèle à un second article contemporain (Cordonier, 2010a). Cette contribution-ci a paru dans les actes du symposium "Évaluation et régulation des compétences professionnelles: entre référentiels et pratiques », qui s'est tenu à l'Université du Québec à Trois-Rivières, les 26 et 27 août 2008 (Cordonier, 2010b).

2. Le référentiel de compétences de la HEP du canton de Vaud qui est examiné ici a été rédigé en 2005 en s'inspirant fortement du référentiel québécois des compétences professionnelles en enseignement (Martinet, Raymond \& Gauthier, 2001) et des référentiels adoptés par les instituts universitaires et hautes écoles pédagogiques de Suisse romande, à commencer par celui de la Faculté de psychologie et des sciences de l'éducation de l'Université de Genève (Perrenoud, 1994). 3. Embrassant une période de 50 ans, le regard historique qui est le nôtre ici ne permet pas d'affiner l'observation jusqu'à prendre en compte les débats et nuances actuels portant sur les concepts d'innovation, de novation, voire d'«énovation». A leurs propos, voir, par exemple, Jacquinot-Delaunay \& Fichez (2008) ou Charlier \& Peraya (2003).

4. Parmi les différentes activités de l'enseignant-expert, nous ne retiendrons ci-après que celle qui l'appelle à certifier l'atteinte par ses étudiants des objectifs des enseignements et modules qu'il dispense.

5. Je remercie chaleureusement mon collègue de m'avoir autorisé à publier cette grille.

6. On prend le mot «transcendantal» dans le sens que lui donne Dany-Robert Dufour: le transcendantal caractérise le monde moderne qui, depuis Kant, a permis de quitter les sujétions de la transcendance. "Le monde transcendantal de Kant correspond en effet à une sortie des déterminations locales [...] 》 (Dufour, 2008, p. 187) des " [...] anciennes assignations métaphysiques du Je » (Dufour, 2008, p. 129), celles des religions ou même de Descartes. Libérée des dogmes anciens, la raison pure est ce centre vide qui suscite l'imagination transcendantale, laquelle fonde l'obligation morale du sujet critique » (Idem).

\section{RÉSUMÉS}

Cette contribution s'intéresse à l'une des qualités centrales de l'enseignant tel qu'il est postulé par les référentiels de compétence: l'expertise telle qu'elle s'exerce dans les activités d'évaluation. S'appuyant sur un protocole de certification utilisé à la Haute école pédagogique (HEP) du canton de Vaud, cette étude de cas tente de définir l'épistémologie du sujet enseignant, dans son rôle d'évaluateur. Ce sujet est-il, comme le veut le sens courant du mot, le sujet de quelque chose, ou, au contraire, une individualité forte, autonome, indépendante ? Endosse-t-il pleinement le jugement évaluatif ou se réfugie-t-il derrière des procédures sophistiquées ? Est-il souverain ou timoré, voire peureux ? Pour y répondre, en sus d'analyser une grille et des critères d'évaluation estimés prototypiques, on recourra à l'histoire des idées et, notamment, au postmodernisme et à certains de ses avatars, directs ou indirects (la raison instrumentale, la notion de dispositif,...). 
INDEX

Mots-clés : enseignant évaluateur, enseignant innovateur, épistémologie du sujet enseignant, postmodernisme, référentiel de compétences, tâches enseignant

\section{AUTEUR}

\section{NOËL CORDONIER}

Haute école pédagogique du canton de Vaud Noel.cordonier@hepl.ch 